\title{
DIMENSION AND FINITE CLOSURE
}

\author{
W. F. GROSS
}

(Received 7 June 1976)

\begin{abstract}
If $\mathfrak{M}$ is a model with dimension and finite closure, then $T(\mathfrak{M})$ is $\boldsymbol{N}_{0}$-categorical. If $\mathfrak{M}$ is atomic, has dimension and finitely many algebraic elements, then $\mathfrak{D}$ has finite closure or a finite basis. If $\mathfrak{W}$ has finite closure, satisfies the Exchange Lemma, and one-one maps between independent subsets are elementary, then $\mathfrak{P}$ has dimension.
\end{abstract}

In Crossley \& Nerode (1974, p. 44), the authors assume that the theories which they treat are $\boldsymbol{N}_{0}$-categorical, but note that it is sufficient, for their purposes, to consider a complete theory $T$ for which each $B_{n}(T)$ is atomistic and every model has finite closure. A large part of their work concerns models with dimension. We show, in Section 1, that for a complete theory $T$ with an infinite model which can be covered by finitely many minimal formulae, in particular with a model with dimension, $T$ must be $\boldsymbol{N}_{0}$-categorical for its model to have finite closure. We also show, in Section 2, that if a model is atomic, has dimension and finitely many algebraic elements, then it has either a finite basis or finite closure.

If $\mathfrak{M}$ has dimension, then $\mathfrak{M}$ satisfies the Exchange Lemma and one-one maps between independent subsets of $\mathfrak{M}$ are elementary (see Propositions 1 and 3). We show, in Section 3, that if $\mathfrak{M}$ has these two properties and finite closure, then $\mathfrak{M}$ has dimension. No form of the axiom of choice is used.

Section 0 gives the notation and conventions we follow, as well as the necessary definitions and propositions from Crossley \& Nerode (1974).

$\mathbf{0}$

Models. For a model, $\mathfrak{M}$, we use $\mathfrak{M}$ to denote the domain of $\mathfrak{M}$, if no ambiguity arises. We use $a, b$, etc. to denote elements of $\mathfrak{M}$ and $A, B$ etc. to

'Sections of this paper were part of a thesis submitted for the degree of Doctor of Philosophy at Monash University and under the supervision of Professor J. N. Crossley. 
denote subsets of $\mathfrak{M} . T(\mathfrak{M})$ denotes the complete theory of $\mathfrak{M}$. We do not assume that the language of a model is countable but we do assume that the language contains a symbol for equality and that $\mathfrak{M}$ is a normal model. Thus we can define in the language quantifiers $\exists^{<k} v_{0} \cdots$ meaning "there exist $<k$ $v_{0} \ldots$ " and $\exists^{k} v_{0} \cdots$ meaning "there exist exactly $k v_{0} \cdots ", \chi\left(v_{0}, \cdots, v_{n}\right)$ will always denote a formula of the language of $\mathfrak{M}$ with all its free variables among $v_{0}, \cdots, v_{n}$. For $a_{0}, \cdots, a_{n} \in \mathfrak{M}$ we write $\mathfrak{M} \vDash \chi\left(v_{0}, \cdots, v_{n}\right)\left[a_{0}, \cdots, a_{n}\right]$ or $\mathfrak{M} \vDash \chi\left[a_{1}, \cdots, a_{n}\right]$ if $a_{0}, \cdots, a_{n}$ satisfies $\chi$ in $\mathfrak{M}$. If $T$ is a complete theory, $B_{n}(T)$ denotes the boolean algebra of equivalence classes of formulae of the language of $T$ with all their free variables among $v_{0}, \cdots, v_{n-1}$, where $\chi\left(v_{0}, \cdots, v_{n-1}\right), \psi\left(v_{0}, \cdots, v_{n-1}\right)$ are equivalent if $T \vdash \forall v_{0}, \cdots, v_{n-1}(\chi \leftrightarrow \psi)$. We use $\chi$ to denote the equivalence class containing $\chi$ as no ambiguity arises. A model $\mathfrak{M}$ is atomic, if for every $n$-tuple $\left(a_{0}, \cdots, a_{n-1}\right)$ of $\mathfrak{M}$ there is an atom $\chi$ of $B_{n}\left(T(\mathfrak{M})\right.$ ) such that $\mathfrak{M} \vDash \chi\left[a_{0}, \cdots, a_{n-1}\right]$. We say $\mathfrak{M}$ is covered by the formulae $\chi_{1}\left(v_{0}\right), \cdots, \chi_{n}\left(v_{0}\right)$ if $\mathfrak{M} \vDash \forall v_{0}\left(\chi_{1} \vee \cdots \vee \chi_{n}\right)$. a is a solution of $\chi\left(v_{0}\right)$ if $\mathfrak{W} F \chi[a]$.

Algebraic Closure. We follow chapters 4 and 6 of Crossley \& Nerode (1974). $a$ is algebraic over $A$ if for some $a_{1}, \cdots, a_{n} \in A, \chi\left(v_{n}, \cdots, v_{n}\right)$ and natural number $k$,

$$
\mathfrak{M} \vDash\left(\exists^{<k} v_{0} X\left(v_{0}, \cdots, v_{n}\right) \& \times\left(v_{0}, \cdots, v_{n}\right)\right)\left[a, a_{1}, \cdots, a_{n}\right] .
$$

$a$ is algebraic if it is algebraic over $\phi$. The algebraic closure of $A, \mathrm{cl} A$, is the set of all elements of $\mathfrak{M}$ algebraic over $A$. Clearly $A \subseteq \mathrm{cl} A . \mathfrak{M}$ has finite closure if $\operatorname{cl} A$ is finite whenever $A$ is finite. $A$ is independent if for all $a \in A$, $a \notin \mathrm{cl}(A \backslash\{a\})$. We write $\left(a_{1}, \cdots, a_{n}\right)$ is independent if $\left\{a_{1}, \cdots, a_{n}\right\}$ is independent and the $a_{i}$ are distinct. $A$ is a basis of $\mathfrak{M}$ if $A$ is independent and $\mathfrak{M}=\mathrm{cl} A . \phi\left(v_{0}\right)$ is a minimal formula for $\mathfrak{M}$ if $\phi$ has infinitely many solutions in $\mathfrak{M}$ and for each $\psi\left(v_{0}, \cdots, v_{n}\right)$ and $a_{1}, \cdots, a_{n} \in \mathfrak{M}$ either $\phi\left(v_{0}\right) \&$ $\psi\left(v_{0}, a_{1}, \cdots, a_{n}\right)$ or $\phi\left(v_{0}\right) \& \neg \psi\left(v_{0}, a_{1}, \cdots, a_{n}\right)$ has finitely many solutions in $\mathfrak{M}$. Clearly if $\phi\left(v_{0}\right)$ is minimal and $\psi\left(v_{0}\right)$ has only finitely many solutions then $\phi \vee \psi$ and $\phi \& \neg \psi$ are minimal. Min $(\mathfrak{M})$ is the set of solutions of minimal formulae. $\mathfrak{M}$ has dimension if for some minimal formula $\phi, \mathfrak{M} \vDash \phi[a]$ for every non-algebraic element, $a$, of $\mathfrak{M}$. If $\mathfrak{M}, \mathfrak{M}^{\prime}$ have the same language $\mathscr{L}$, $A \subseteq \mathfrak{M}, A^{\prime} \subseteq \mathfrak{M}^{\prime}$ and $p: A \rightarrow A^{\prime}$, then $p$ is an elementary monomorphism if for all $a_{0}, \cdots, a_{n} \in A$ and for all $\chi\left(v_{10}, \cdots, v_{n}\right) \in \mathscr{L}$

$$
\mathfrak{M} \vDash \chi\left[a_{0}, \cdots, a_{n}\right] \text { if and only if } \mathfrak{D}^{\prime} \vDash \chi\left[p a_{0}, \cdots, p a_{n}\right]
$$

( $p$ is one-one as $\mathscr{L}$ contains equality and $\mathfrak{D}\}$ is a normal model).

We use the following propositions. 
Proposition 1. (Crossley \& Nerode (1974), Lemma 6.4(ib)). (Exchange Lemma) For any model, $\mathfrak{M}$, if $\left\{a_{1}, \cdots, a_{n}\right\} \subseteq \mathfrak{M}$ is independent but $\left\{a_{1}, \cdots, a_{n+1}\right\}$ is not, and $a_{n+1} \in \operatorname{Min}(\mathscr{M})$, then $a_{n+1} \in \operatorname{cl}\left\{a_{1}, \cdots, a_{n}\right\}$.

Proposition 2. (Crossley \& Nerode (1974), Lemma 6.4(ii)). For any model $\mathfrak{M}$, suppose $A, B \subseteq \operatorname{Min}\left(\mathfrak{M}_{)}\right), \mathrm{cl} A \subseteq \mathrm{cl} B$ and $A$ is independent. Then

(a) $\operatorname{card} A \leqq \operatorname{card} B$

(b) there is a subset $B_{0}$ of $B$ such that $A \cup B_{0}$ is independent and $\operatorname{cl}\left(A \cup B_{0}\right)=\operatorname{cl} B$.

An obvious and trivial modification of the proof of Crossley \& Nerode (1974), Lemma 6.9, gives:

Proposition 3. Let $\mathfrak{M}, \mathfrak{M}^{\prime}$ be models of a complete theory $T, A \subseteq$ Min $(\mathfrak{M}), B \subseteq \mathfrak{M}^{\prime}$ independent sets and $p: A \rightarrow B$ a one-one map such that for $a \in A$ there is some minimal formula, $\phi\left(v_{0}\right)$ for $\mathfrak{M}$ such that $\mathfrak{M} \vDash \phi[a]$ and $\mathfrak{M}^{\prime} \vDash \phi[p(a)]$. Then $p$ is an elementary monomorphism.

1

We can prove our first result immediately.

THEOREM 4. Suppose a complete theory $T$ has an infinite model $\mathfrak{M}$ with finite closure which is covered by minimal formulae $\phi_{1}, \cdots, \phi_{n}$. Then $B_{m}(T)$ is finite for all $m$ and $T$ is $\boldsymbol{N}_{0}$-categorical.

Proof. We may assume that $\mathfrak{M} \models \wedge_{i \neq j} \forall v_{0} \neg\left(\phi_{i}\left(v_{0}\right) \& \phi_{j}\left(v_{0}\right)\right)$. For if, for $i \neq j, \mathfrak{M} \models\left(\phi_{i} \& \phi_{j}\right)[a]$ for infinitely many $a$ then replace $\phi_{i}$ (say) with $\phi_{i} \vee \phi_{i}$ and delete $\phi_{j}$. Now $\mathfrak{M} \vDash\left(\phi_{i} \& \neg \phi_{j}\right)[a]$ for finitely many $a$, so $\phi_{j} \vee\left(\phi_{i} \& \neg \phi_{i}\right)$ (i.e. $\left.\phi_{i} \vee \phi_{j}\right)$ is again minimal. If $\mathfrak{M} \vDash\left(\phi_{i} \& \phi_{i}\right)$ [a] for finitely many $a$ and $i<j$ replace $\phi_{j}$ with $\phi_{j} \& \neg \phi_{i}$, which is again minimal. In both cases the new $\phi$ 's cover $\mathfrak{M}$, so a simple induction validates the assumption.

Let $D_{i}=\left\{a \in \mathfrak{M}: \mathfrak{N}\left\{\vDash \phi_{i}[a]\right\}\right.$. By the definition of a minimal formula each $D_{i}$ is infinite.

Suppose $m \in \omega$. Then there is an independent subset $C$ of $\mathfrak{M}$ such that card $C \cap D_{i}=m$ for all $i$, for if not, let $r$ be the least $m$ for which it fails. Then $r>0$, and there is an independent $C^{\prime}$ such that card $C^{\prime} \cap D_{i}=r-1$. As $\operatorname{cl} C^{\prime}$ is finite and $D_{1}$ is infinite, there is $c_{1} \in D_{1}$ such that $c_{1} \notin \mathrm{cl} C^{\prime}$. So by Proposition $1, C^{\prime} \cup\left\{c_{1}\right\}$ is independent. Thus we can construct by induction $C^{\prime \prime}$ such that card $C^{\prime \prime} \cap D_{i}=r$ for $i=1, \cdots, n$, contradicting the choice of $r$.

If $\chi \in B_{m}(T), \chi \neq 0$, then $T \vdash \exists v_{1}, \cdots, v_{m} \chi\left(v_{1}, \cdots, v_{m}\right)$. So there are $a_{1}, \cdots, a_{m} \in \mathfrak{M}$ such that $\mathfrak{M} \vDash \chi\left[a_{1}, \cdots, a_{m}\right]$.

By Proposition 2 there is an independent set $A=\left\{a_{1}^{\prime}, \cdots, a_{m}^{\prime}\right\} \subseteq$ 
$\left\{a_{1}, \cdots, a_{m}\right\}$ such that $\operatorname{cl}\left\{a_{1}, \cdots, a_{m}\right\}=\operatorname{cl} A$. Thus $\left\{a_{1}, \cdots, a_{m}\right\} \subseteq \operatorname{cl} A$. As card $D_{i} \cap A \leqq m$ there is a one-one map $p: A \rightarrow C$ which satisfies the hypothesis of Proposition 3 and so is elementary.

As $\left\{a_{1}, \cdots, a_{m}\right\} \subseteq \mathrm{cl} A$, for $i=1, \cdots, m$, there are formulae $\psi_{i}\left(v_{0}, \cdots, v_{m}\right)$, $\sigma_{i}\left(v_{0}, \cdots, v_{m}\right)$ and natural numbers $k_{i}$ such that

$$
\sigma_{i}\left(v_{0}, \cdots, v_{m}\right)=\left(\psi_{i}\left(v_{0}, \cdots, v_{m}\right) \& \exists^{<k_{i}} v_{0} \psi_{i}\left(v_{1}, \cdots, v_{m}\right)\right)
$$

and

$$
\mathfrak{M} \vDash \sigma_{i}\left[a_{i}, a_{i}^{\prime}, \cdots, a_{m}^{\prime}\right] .
$$

Hence $\mathfrak{R} \vDash\left(\exists u_{1}, \cdots, u_{m}\left(\chi\left(u_{1}, \cdots, u_{m}\right) \& \wedge_{i=1}^{m} \sigma_{i}\left(u_{i}, v_{1}, \cdots, v_{m}\right)\right)\right)\left[a_{1}^{\prime}, \cdots, a_{m}^{\prime}\right]$ and so

$$
\mathfrak{R} \vDash\left(\exists u_{1}, \cdots, u_{m}\left(\chi\left(u_{1}, \cdots, u_{m}\right) \& \wedge_{i=1}^{m} \sigma_{i}\left(u_{i}, v_{1}, \cdots, v_{m}\right)\right)\right)\left[p\left(a_{i}^{\prime}\right), \cdots, p\left(a_{m^{\prime}}^{\prime}\right)\right] .
$$

As $p\left(a_{i}^{\prime}\right) \in C$, there exist $c_{1}, \cdots, c_{m} \in \operatorname{cl} C$ such that $\mathfrak{M}^{\prime} \vDash \chi\left[c_{1}, \cdots, c_{m}\right]$.

The map $q: B_{m}(T) \rightarrow \mathscr{P}\left((\mathrm{cl} C)^{m}\right)$ given by

$$
q(\chi)=\left\{\left(c_{1}, \cdots, c_{m}\right) \in(\mathrm{cl} C)^{m}: \mathscr{D}_{i} \vDash \chi\left[c_{1}, \cdots, c_{m}\right]\right\}
$$

is one-one, for suppose $\chi_{1}, \chi_{2} \in B_{m}(T)$ and $\chi_{1} \neq \chi_{2}$. Then we may assume $\chi_{1} \& \neg \chi_{2} \neq 0$. So by the above, there are $c_{1}, \cdots, c_{m} \in C$ such that $\mathfrak{M} \vDash \chi_{1} \& \neg \chi_{2}\left[c_{1}, \cdots, c_{m}\right]$ and therefore $q\left(\chi_{1}\right) \neq q\left(\chi_{2}\right)$. But $\mathscr{P}\left((\mathrm{cl} C)^{m}\right)$ is finite as $\mathrm{cl} C$ is, whence $B_{m}(T)$ is finite.

So by Ryll-Nardzewski (1959), $T$ is $\boldsymbol{N}_{11}$-categorical. We note that this direction of Ryll-Nardzewski's proof does not require the axiom of choice.

Regarding the converse of Theorem 4 , if $\mathfrak{M} \vDash T$ and $B_{m}(T)$ is finite for all $m$, indeed just for $m=1$, then $\mathfrak{M}$ can have at most finitely many minimal formulae, as it has only finitely many inequivalent 1-place formulae. However $(Q, \leqq)$ is a model of an $\boldsymbol{N}_{0}$-categorical theory and has no minimal formulae.

Corollary 5. If $T$ is a complete theory with a model $\mathfrak{M}$ with dimension and finite closure, then $B_{m}(T)$ is finite for each $m$ and $T$ is $\boldsymbol{N}_{n}$-categorical.

Proof. As $\mathfrak{M}$ has finitely many algebraic elements, $v_{0}=v_{0}$ is a minimal formula which covers $\mathfrak{M}$.

COROLlary 6. If $T$ is a complete theory with a model $\mathfrak{M}$ with dimension and finite closure then every model $\mathfrak{M}$ of $T$ has dimension and finite closure.

Proof. By Corollary $5, B_{m}(T)$ is finite for each $m$ and so by Crossley \& Nerode (1974), Lemma 5.9, $\mathfrak{R}$ has finite closure. Furthermore $\mathfrak{R}$ is atomic. 
As $\mathfrak{M}$ has dimension and finite closure, $v_{0}=v_{0}$ is a minimal formula for $\mathfrak{M}$. We will show $v_{0}=v_{0}$ is a minimal formula for $\mathfrak{R}$.

Let $a_{1}, \cdots, a_{n} \in \mathfrak{N}$ and $\chi\left(v_{0}, \cdots, v_{n}\right)$ be any formula. Let $\psi\left(v_{0}, \cdots, v_{n-1}\right)$ be the atom satisfied by $a_{1}, \cdots, a_{n}$ and let $b_{1}, \cdots, b_{n} \in \mathfrak{M}$ satisfy $\psi$. As $v_{0}=v_{0}$ is a minimal formula for $\mathfrak{M}$,

$$
\mathfrak{M} \vDash \exists^{<k} v_{0} \sigma\left(v_{0}, \cdots, v_{n}\right)\left[b_{1}, \cdots, b_{n}\right],
$$

for some finite $k$, where $\sigma$ is $\chi$ or $\neg \chi$. Hence

$$
T \vdash \forall v_{1}, \cdots, v_{n}\left(\psi\left(v_{1}, \cdots, v_{n}\right) \rightarrow \exists^{<k} v_{0} \sigma\left(v_{0}, \cdots, v_{n}\right)\right)
$$

as $\psi$ is an atom and so

$$
\mathfrak{N} \vDash \exists^{<k} v_{0} \sigma\left(v_{0}, \cdots, v_{n}\right)\left[a_{1}, \cdots, a_{n}\right] .
$$

So $v_{0}=v_{0}$ is minimal for $\mathfrak{N}$ whence $\mathfrak{R}$ has dimension.

2

We first prove a theorem from which our second claim follows readily:

THEOREM 7. Suppose $\mathfrak{M}$ is an atomic model of a complete theory $T$ and $\phi\left(v_{0}\right)$ is a minimal formula for $\mathfrak{M}$. Then for all $n$ such that $\mathfrak{M}$ contains an independent set with $\geqq n+1$ solutions of $\phi$, there is a formula $\rho_{n+1}$, an atom of $B_{n+1}(T)$, such that for any model $\mathfrak{M}^{\prime}$ of $T$ :

$\mathfrak{M}^{\prime} \vDash \rho_{n+1}\left[a_{0}^{\prime}, \cdots, a_{n}^{\prime}\right]$ if and only if

$$
\left(a_{0}^{\prime}, \cdots, a_{n}^{\prime}\right) \quad \text { is independent and } \quad \mathfrak{M}^{\prime} \vDash \phi\left[a_{i}^{\prime}\right] \quad i=0, \cdots, n \text {. }
$$

Proof. Suppose $\left(a_{0}, \cdots, a_{n}\right) \subseteq \mathfrak{M}$ is independent and $\mathfrak{M} \vDash \phi\left[a_{i}\right] i=$ $0, \cdots, n$. Then the $a_{i}$ are distinct. As $\mathfrak{M}$ is atomic, there is an atom $\rho_{n+1}$ of $B_{n+1}(T)$ such that $\mathfrak{M} \vDash \rho_{n+1}\left[a_{0}, \cdots, a_{n}\right]$. We show that it has the desired property.

Suppose $\left(a_{0}^{\prime}, \cdots, a_{n}^{\prime}\right) \subseteq \mathfrak{M}^{\prime}$ is independent and $\mathfrak{M}^{\prime} \vDash \phi\left[a_{i}^{\prime}\right] \quad i=0, \cdots, n$. Then by Proposition 3, $p: a_{i} \mapsto a_{i}^{\prime}$ is elementary whence $\mathfrak{M}^{\prime} \vDash \rho_{n+1}\left[p\left(a_{0}\right), \cdots, p\left(a_{n}\right)\right]$ which is precisely $\mathfrak{M}^{\prime} \vDash \rho_{n+1}\left[a_{0}^{\prime}, \cdots, a_{n}^{\prime}\right]$.

Conversely, suppose $\mathfrak{M}^{\prime} \models \rho_{n+1}\left[a_{0}^{\prime}, \cdots, a_{n}^{\prime}\right]$ and $\left(a_{0}^{\prime}, \cdots, a_{n}^{\prime}\right)$ is dependent. We may assume, without loss of generality, that $a_{0}^{\prime} \in \operatorname{cl}\left\{a_{1}^{\prime}, \cdots, a_{n}^{\prime}\right\}$. So there is a formula $\psi\left(v_{0}, \cdots, v_{n}\right)$ and natural number $k$, such that

$$
\mathfrak{M}^{\prime} \models\left(\psi\left(v_{0}, \cdots, v_{n}\right) \& \exists^{<k} v_{0} \psi\left(v_{0}, \cdots, v_{n}\right)\right)\left[a_{0}^{\prime}, \cdots, a_{n}^{\prime}\right] .
$$

So

$$
T \vdash \exists v_{0}, \cdots, v_{n}\left(\rho_{n+1} \& \psi \& \exists^{<k} v_{0} \psi\right)
$$


But $\rho_{n+1}$ is an atom of $B_{n+1}(T)$.

So

$$
T \vdash \forall v_{0}, \cdots, v_{n}\left(\rho_{n+1} \rightarrow\left(\psi \& \exists^{<k} v_{0} \psi\right)\right)
$$

But $\quad \mathfrak{M} \models \rho_{n+1}\left[a_{0}, \cdots, a_{n}\right]$, so $\mathfrak{M} \vDash\left(\psi \& \exists^{<k} v_{0} \psi\right)\left[a_{0}, \cdots, a_{n}\right]$.

Hence $a_{0} \in \operatorname{cl}\left\{a_{1}, \cdots, a_{n}\right\}$ which contradicts the independence of $\left(a_{0}, \cdots, a_{n}\right)$. So $\left(a_{0}^{\prime}, \cdots, a_{n}^{\prime}\right)$ is independent.

It remains to show that $\mathfrak{M}^{\prime} \models \phi\left[a_{i}^{\prime}\right] i=0, \cdots, n$.

$$
\mathfrak{M} \vDash\left(\rho_{n+1} \& \underset{i=0}{\wedge} \phi\left(v_{i}\right)\right)\left[a_{0}, \cdots, a_{n}\right] .
$$

Hence $T \vdash \forall v_{0}, \cdots, v_{n}\left(\rho_{n+1} \rightarrow \wedge_{i=0}^{n} \phi\left(v_{i}\right)\right)$ as $\rho_{n+1}$ is an atom of $B_{n+1}(T)$, and so $\mathfrak{M}^{\prime} \vDash \wedge_{i=0}^{n} \phi\left(v_{i}\right)\left[a_{0}^{\prime}, \cdots, a_{n}^{\prime}\right]$ as $\mathfrak{M}^{\prime} \vDash \rho_{n+1}\left[a_{i}^{\prime}, \cdots, a_{n}^{\prime}\right]$.

Corollary 8. Suppose $\mathfrak{M}$ is an atomic model of a complete theory $T$, $\phi\left(v_{0}\right)$ is a minimal formula and $D=\{a: \mathfrak{M} \vDash \phi[a]\}$. If there are arbitrarily large finite independent subsets of $D$, then for any finite $A \subseteq D, D \cap \operatorname{cl} A$ is finite.

Proof. Suppose for some $A \subseteq D$, that $A$ is finite but $D \cap \operatorname{cl} A$ is infinite. Let $A=\left\{a_{1}, \cdots, a_{n}\right\}$. We may assume that $A$ is independent, for by Proposition 2, there is an independent $A^{\prime} \subseteq A$ such that $\operatorname{cl} A^{\prime}=\operatorname{cl} A$ (No choice is needed as $A$ is finite). By Theorem 7 and the hypothesis there is an atom of $B_{n+1}(T), \rho_{n+1}\left(v_{0}, \cdots, v_{n}\right)$, such that for $d_{i} \in D, \mathfrak{M} \models \rho_{n+1}\left[d_{0}, \cdots, d_{n}\right]$ if and only if $\left(d_{0}, \cdots, d_{n}\right)$ is independent. By Proposition 1 , if $\left(d_{1}, \cdots, d_{n}\right)$ is independent, $\mathfrak{M} \models \rho_{n+1}\left[d_{0}, \cdots, d_{n}\right]$ if and only if $d_{0} \notin \operatorname{cl}\left\{d_{1}, \cdots, d_{n}\right\}$.

As $D \cap \mathrm{cl} A$ is infinite then $\phi\left(v_{0}\right) \& \neg \rho_{n+1}\left(v_{0}, a_{1}, \cdots, a_{n}\right)$ has infinitely many solutions in $\mathfrak{M}$, and as $\phi$ is minimal, $\phi\left(v_{0}\right) \& \rho_{n+1}\left(v_{0}, a_{1}, \cdots, a_{n}\right)$ has finitely many solutions, $d_{1}, \cdots, d_{k}$ say.

Hence $\quad D \subseteq \operatorname{cl}\left\{a_{1}, \cdots, a_{n}, d_{1}, \cdots, d_{k}\right\} \quad$ and $\quad\left\{a_{1}, \cdots, a_{n}, d_{1}, \cdots, d_{k}\right\} \subseteq$ $\operatorname{Min}(\mathfrak{M})$. So if $\left\{a_{1}^{\prime}, \cdots, a_{m}^{\prime}\right\} \subseteq D$ is independent, by Proposition 2,

$$
m=\operatorname{card}\left\{a_{1}^{\prime}, \cdots, a_{m}^{\prime}\right\} \leqq \operatorname{card}\left\{a_{1}, \cdots, a_{n}, d_{1}, \cdots, d_{k}\right\} \leqq n+k
$$

which contradicts the hypothesis of the corollary.

Hence $D \cap \operatorname{cl} A$ is finite for all finite $A \subseteq D$.

The main result of this section is:

COROLLARY 9. If $\mathfrak{M}$ is an atomic model with dimension and finitely many algebraic elements then $\mathfrak{M}$ has a finite basis or finite closure. 
Proof. The following are clear, as is the deduction of Corollary 9 from them and Corollary 8.

If $\mathfrak{M}$ has dimension and $\mathrm{cl} \phi$ is finite there is a minimal formula $\phi$ for which $D=\mathfrak{M}$. If $\mathfrak{M}$ does not have a finite basis then it has arbitrarily large independent subsets.

We can find atomic models with dimension and finitely many algebraic elements with a finite basis but not finite closure $((Z, S)$ where $S(n)=n+1)$ and with no finite basis but finite closure $((N,=))$. Models with a finite basis and finite closure are finite and so do not have dimension, as we require a minimal formula to have infinitely many solutions.

If we do not assume that $\mathfrak{M}$ is atomic, then Corollary 9 is false. If we take $\mathfrak{M}=\left(V,+, f_{\lambda}\right)_{\lambda \in F}$ where $V$ is an infinite dimensional vector space over an infinite field $F$, and $f_{\lambda}: v \mapsto \lambda v$ is a unary function, then $\mathfrak{M}$ has dimension but neither a finite basis nor finite closure, as algebraic closure is closure in the usual vector space sense. $\mathfrak{M}$ is not atomic, for if $\left\{a_{0}, \cdots, a_{n}\right\}$ is independent, $\mathfrak{M} \vDash a_{0} \neq \lambda_{1} a_{1}+\cdots+\lambda_{n} a_{n}$ for all $\lambda_{1}, \cdots, \lambda_{n} \in F$, whereas $B_{n+1}(T)$ is generated by $\left\{\lambda_{0} v_{0}=\lambda_{1} v_{1}+\cdots+\lambda_{n} v_{n}: \lambda_{i} \in F\right\}$ as $T(\mathfrak{M})$ admits elimination of quantifiers. Hence there is no atom satisfied by $\left(a_{0}, \cdots, a_{n}\right)$.

Combining Corollaries 5 and 9 we obtain:

COROLLARY 10. If $T$ is a complete theory with an atomic model with dimension but no finite basis and finitely many algebraic elements then $T$ is $\boldsymbol{N}_{0}$-categorical.

THEOREM 11. Suppose Mi has the following properties.

(1) $\mathfrak{M}$ has finite closure.

(2) If $\left\{a_{1}, \cdots, a_{n}\right\} \subseteq \mathfrak{M}$ is independent and $\left\{a_{1}, \cdots, a_{n+1}\right\}$ is not, then $a_{n+1} \in \operatorname{cl}\left\{a_{1}, \cdots, a_{n}\right\}$. (M satisfies the Exchange Lemma).

(3) If $A, B \subseteq \mathfrak{M}$ are independent and $p: A \rightarrow B$ is one-one, then $p$ is elementary.

Then $\mathfrak{M}$ has dimension.

ProOF. We prove the following by induction on $n$ :

(4) If $\left\{a_{1}, \cdots, a_{m}\right\}$ is independent, $b_{1}, \cdots, b_{n} \in \operatorname{cl}\left\{a_{1}, \cdots, a_{m}\right\}$ then there exist $c_{1}, \cdots, c_{p}$ such that $\left\{a_{1}, \cdots, a_{m}, c_{1}, \cdots, c_{p}\right\}$ is independent and for any formula $\psi\left(v_{1}, \cdots, v_{m+n+p+1}\right)$ and for any $d_{1}, d_{2} \notin \operatorname{cl}\left\{a_{1}, \cdots, a_{m}, c_{1}, \cdots, c_{p}\right\}$,

$$
\begin{aligned}
& \mathfrak{M} \vDash \psi\left[a_{1}, \cdots, a_{m}, b_{1}, \cdots, b_{n}, c_{1}, \cdots, c_{p}, d_{1}\right] \text { if, and only if, } \\
& \mathfrak{M} \vDash \psi\left[a_{1}, \cdots, a_{m}, b_{1}, \cdots, b_{n}, c_{1}, \cdots, c_{p}, d_{2}\right] .
\end{aligned}
$$


Suppose $n=0$. If $d_{1}, d_{2} \notin \operatorname{cl}\left\{a_{1}, \cdots, a_{m}\right\}$ then, by (2), $\left\{a_{1}, \cdots, a_{m}, d_{1}\right\}$ and $\left\{a_{1}, \cdots, a_{m}, d_{2}\right\}$ are independent, and (4) holds by (3).

Suppose (4) holds for some $n$ and $b_{n+1} \in \operatorname{cl}\left\{a_{1}, \cdots, a_{m}\right\}$. Then $b_{n+1} \in$ $\operatorname{cl}\left\{a_{1}, \cdots, a_{m}, b_{1}, \cdots, b_{n}, c_{1}, \cdots, c_{p}\right\}$ and there is a formula $\chi_{0}\left(v_{0}, \cdots, v_{m+n+p}\right)$ and a natural number $k_{0} \geqq 1$, such that

$$
\mathfrak{M} F \chi_{0} \& \exists^{k_{0}} v_{0} \chi_{0}\left[b_{n+1}, a_{1}, \cdots, a_{m}, b_{1}, \cdots, b_{n}, c_{1}, \cdots, c_{p}\right] .
$$

We construct sequences $k_{0}>k_{1}>\cdots>k_{q} \geqq 1, \chi_{0}, \cdots, \chi_{q}, c_{p+1}, \cdots, c_{p+q}$, such that for any formula $\psi\left(v_{1}, \cdots, v_{m+n+p+q+2}\right)$ and $d_{1}, d_{2} \notin \operatorname{cl}\left\{a_{1}, \cdots, a_{m}\right.$, $\left.c_{1}, \cdots, c_{p+q}\right\}$

$$
\begin{aligned}
& \mathfrak{M}_{i}=\psi\left[a_{1}, \cdots, a_{m}, b_{1}, \cdots, b_{n+1}, c_{1}, \cdots, c_{p+q}, d_{1}\right] \text { if, and only if, } \\
& \mathfrak{M}_{\models} \models \psi\left[a_{1}, \cdots, a_{m}, b_{1}, \cdots, b_{n+1}, c_{1}, \cdots, c_{p+q}, d_{2}\right] .
\end{aligned}
$$

If (5) holds with $q=0$, we are done. If not, there is a formula $\psi\left(v_{1}, \cdots, v_{m+n+p+2}\right)$ and $d_{1}, d_{2} \notin \operatorname{cl}\left\{a_{1}, \cdots, a_{m}, c_{1}, \cdots, c_{p}\right\}$ such that

$$
\mathfrak{M} \vDash \psi\left[a_{1}, \cdots, a_{m}, b_{1}, \cdots, b_{n+1}, c_{1}, \cdots, c_{p}, d_{1}\right]
$$

and

$$
\mathfrak{M} \vDash \neg \psi\left[a_{1}, \cdots, a_{m}, b_{1}, \cdots, b_{n+1}, c_{1}, \cdots, c_{p}, d_{2}\right] .
$$

Put $c_{p+1}=d_{1}$ and put

$$
\begin{aligned}
\chi_{1}\left(v_{0}, \cdots, v_{m+n+p+2}\right) & \\
& =\chi_{0}\left(v_{0}, \cdots, v_{m+n+p}\right) \& \psi\left(v_{1}, \cdots, v_{m+n}, v_{0}, v_{m+n+1}, \cdots, v_{m+n+p+2}\right) .
\end{aligned}
$$

By (2), $\left\{a_{1}, \cdots, a_{m}, c_{1}, \cdots, c_{p+1}\right\}$ is independent. Clearly

$$
\mathfrak{M} \vDash \chi_{1}\left[b_{n+1}, a_{1}, \cdots, a_{m}, b_{1}, \cdots, b_{n}, c_{1}, \cdots, c_{p+1}\right] .
$$

And

$$
\mathfrak{M} \vDash \exists v_{0}\left(\chi_{0} \& \neg \chi_{1}\right)\left[a_{1}, \cdots, a_{m}, b_{1}, \cdots, b_{n}, c_{1}, \cdots, c_{p+1}\right]
$$

for suppose otherwise. Then

$$
\mathfrak{M} \models \forall v_{0}\left(\chi_{0} \rightarrow \chi_{1}\right)\left[a_{1}, \cdots, a_{m}, b_{1}, \cdots, b_{n}, c_{1}, \cdots, c_{p}, d_{1}\right]
$$

whence, by (4),

$$
\mathfrak{M} \models \forall v_{0}\left(\chi_{0} \rightarrow \chi_{1}\right)\left[a_{1}, \cdots, a_{m}, b_{1}, \cdots, b_{n}, c_{1}, \cdots, c_{p}, d_{2}\right] .
$$

But $\mathfrak{M} \vDash \chi_{0}\left[b_{n+1}, a_{1}, \cdots, a_{m}, b_{1}, \cdots, b_{n}, c_{1}, \cdots, c_{p}\right]$ and so

$$
\mathfrak{M} \models \chi_{1}\left[b_{n+1}, a_{1}, \cdots, a_{m}, b_{1}, \cdots, b_{n}, c_{1}, \cdots, c_{p}, d_{2}\right]
$$

and therefore 


$$
\mathfrak{M}_{\models}=\psi\left[a_{1}, \cdots, a_{m}, b_{1}, \cdots, b_{n+1}, c_{1}, \cdots, c_{p}, d_{2}\right]
$$

which contradicts (7). Thus (8) holds and

$$
\mathfrak{M} \vDash \exists^{k_{1}} v_{0} \chi_{1}\left[v_{0}, a_{1}, \cdots, a_{m}, b_{1}, \cdots, b_{n}, c_{1}, \cdots, c_{p+1}\right]
$$

where $1 \leqq k_{1}<k_{0}$.

We can choose $\chi_{i}, c_{i}$ is a similar fashion until (5) holds, which is when (4) holds for $n+1$.

Thus (4) holds for all $n$.

Now suppose $b_{1}, \cdots, b_{n} \in \mathfrak{M}$ and $\psi\left(v_{0}, \cdots, v_{n}\right)$ is any formula. Using (2), we can choose $a_{1}, \cdots, a_{m} \in\left\{b_{1}, \cdots, b_{n}\right\}$ such that $\left\{a_{1}, \cdots, a_{m}\right\}$ is independent and $b_{1}, \cdots, b_{n} \in \operatorname{cl}\left\{a_{1}, \cdots, a_{m}\right\}$. By (4), there exist $c_{1}, \cdots, c_{p}$ such that for all $\chi\left(v_{0}, \cdots, v_{n}\right)$ and $d_{1}, d_{2} \notin\left\{a_{1}, \cdots, a_{m}, c_{1}, \cdots, c_{p}\right\} \mathfrak{M} \models \chi\left[d_{1}, b_{1}, \cdots, b_{n}\right]$ if, and only if $\mathfrak{M} \vDash \chi\left[d_{2}, b_{1}, \cdots, b_{n}\right]$.

If $\psi\left(v_{0}, b_{1}, \cdots, b_{n}\right)$ has infinitely many solutions in $\mathfrak{M}$, then $\mathfrak{M} \vDash \psi\left[d, b_{1}, \cdots, b_{n}\right] \quad$ for $\quad$ some $\quad d \notin \operatorname{cl}\left\{a_{1}, \cdots, a_{m}, c_{1}, \cdots, c_{p}\right\} \quad$ as $\operatorname{cl}\left\{a_{1}, \cdots, a_{m}, c_{1}, \cdots, c_{p}\right\}$ is finite by (1). Hence $\mathfrak{M} \vDash \psi\left[d, b_{1}, \cdots, b_{n}\right]$ for all $d \notin \operatorname{cl}\left\{a_{1}, \cdots, a_{m}, c_{1}, \cdots, c_{p}\right\}$ and $\mathfrak{M} \vDash \neg \psi\left[d, b_{1}, \cdots, b_{n}\right]$ for at most $d \in$ $\operatorname{cl}\left\{a_{1}, \cdots, a_{m}, c_{1}, \cdots, c_{p}\right\}$. Thus $\neg \psi\left(v_{0}, b_{1}, \cdots, b_{n}\right)$ has finitely many solutions, and so $v_{0}=v_{0}$ is a minimal formula.

Therefore $\mathfrak{M}$ has dimension.

Conditions (2) and (3) are not sufficient for $\mathfrak{M}$ to have dimension. Consider the model $\mathfrak{Y}=(Z \times Z,<, S)$ where

$$
\left(n_{1}, m_{1}\right)<\left(n_{2}, m_{2}\right) \text { if } n_{1}=n_{2} \text { and } m_{1}<m_{2}
$$

and

$$
S((n, m))=(n, m+1) .
$$

It is easy to see the following:

(a) $\operatorname{cl}\left\{\left(n_{1}, m_{1}\right), \cdots,\left(n_{1}, m_{1}\right)\right\}=\left\{n_{1}, \cdots, n_{l}\right\} \times Z$.

(b) $\left\{\left(n_{1}, m_{1}\right), \cdots,\left(n_{l}, m_{l}\right)\right\}$ is independent if and only if $n_{1}, \cdots, n_{l}$ are distinct, and therefore (2) holds.

(c) If $A, B \subseteq \mathfrak{N}$ are finite and independent and $p: A \rightarrow B$ is one-one, then $p$ extends to an automorphism of $\mathfrak{N}$ and so is elementary. Therefore (3) holds.

(d) $\mathfrak{N}$ has no algebraic elements and $v_{0}<a_{1}$, ᄀ $v_{0}<a_{1}$ both have infinitely many solutions in $\mathfrak{N}$. Thus $\mathfrak{N}$ does not have dimension.

By Corollary 5 , if $\mathfrak{M}$ satisfies (1), (2) and (3), then $B_{n}(T(\mathfrak{M}))$ is finite for each $n$, and so $\mathfrak{M}$ is atomic. However Theorem 11 does not hold if we replace (1) by " $\mathfrak{M}$ is atomic", for the model $\mathfrak{R}$ provides a counter-example. 


$$
\begin{aligned}
\psi & \left(v_{11}, \cdots, v_{1 k_{1}}, \cdots, v_{t 1}, \cdots, v_{i k_{1}}\right) \\
& =\wedge_{i_{1} \neq i_{2}}\left(\neg\left(v_{i_{1} 1}<v_{i_{2} 1}\right) \& \neg\left(v_{i_{2} 1}<v_{i_{1} 1}\right) \& \wedge_{i=1, \cdots, l} \wedge_{i_{2}<j_{1}} v_{i j_{1}}=S^{j_{2}-j_{2}}\left(v_{i_{2}}\right)\right)
\end{aligned}
$$

is a formula satisfied by

$$
\left(\left(n_{1}, m_{11}\right), \cdots,\left(n_{1}, m_{1 k_{1}}\right), \cdots,\left(n_{l}, m_{11}\right), \cdots,\left(n_{l}, m_{k l}\right)\right)
$$

where $n_{1}, \cdots, n_{l}$ are distinct, and is an atom, as can be seen by extending the map $a_{i j} \mapsto a_{i j}^{\prime}$, where $\mathfrak{N} \vDash \psi\left(a_{11}, \cdots, a_{l k_{1}}\right)$ and $\mathfrak{N} \vDash \psi\left(a_{11}^{\prime}, \cdots, a_{i k_{i}}^{\prime}\right)$, to an automorphism of $\mathfrak{N}$.

\section{References}

J. N. Crossley and Anil Nerode (1974), Combinatorial Functors (Springer-Verlag, 1974).

C. Ryll-Nardzewski (1959), 'On theories categorical in power $\boldsymbol{N}^{\prime}$, Bull. Acad. Polon. Sci. Sér. Sci.

Math. Astron. Phys. 7, 545-548.

Department of Mathematics,

University of Tasmania,

Hobart, Australia. 